\title{
Effective medium theories for composite optical materials in spectral ranges of weak absorption: the case of $\mathrm{Nb}_{2} \mathrm{O}_{5^{-}}$ $\mathrm{SiO}_{2}$ mixtures
}

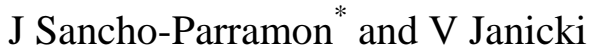 \\ Institute Ruđer Bošković, Bijenička 54, 10000 Zagreb, Croatia
}

This is an author-created, un-copyedited version of an article accepted for publication in Journal of Physics D: Applied Physics. IOP Publishing Ltd is not responsible for any errors or omissions in this version of the manuscript or any version derived from it. The definitive publisher authenticated version is available online at $\underline{10.1088 / 0022-3727 / 41 / 21 / 215304}$

E-mail: j.sancho.parramon@gmail.com

\begin{abstract}
The validity of effective medium theories (EMTs) for mixtures of dielectric materials in weak absorption regions is studied. Based on the Bergman spectral representation, it is possible to show that for any EMT the absorption properties of a mixture consist basically of scaling of the absorption properties of the material with highest absorption. The real part of the dielectric function remains unaffected by the absorption properties. Thin films consisting of $\mathrm{Nb}_{2} \mathrm{O}_{5}-\mathrm{SiO}_{2}$ mixtures are characterized using optical measurements and the results are compared with the calculations of EMTs. The large discrepancies between the absorption properties observed experimentally and those calculated using EMTs are justified by the failure of these theories to predict a compositional dependence of relevant structural parameters, as the band gap energy or the width of localized states. This failure, however, affects the calculation of the refractive index in the weak absorption regions in a less significant degree.
\end{abstract}

PACS codes: 78.66.Nk, 78.20.Bh, 78.20.Ci

\section{Introduction}

One of the most relevant of multiple applications of composite or mixed thin films is the possibility to obtain materials with tailored optical constants. The capacity to engineer refractive index has enabled the development of technologically relevant concepts like gradient refractive index coatings [1] and hybrid coatings [2] in which the composition of the coating, and thus the refractive index, is changed through its thickness. Such coatings show significant advantages in respect to classical multilayer stacks, like reduced scattering losses, improved mechanical properties and higher resistance against radiation [3-6]. Consequently, the theoretical design and manufacturing process of this kind of coatings are subjects of current research [7,8].

In order to calculate the optical properties of gradient refractive index and hybrid coatings, it is necessary to know the dependence of optical constants with mixture composition. The most common approach to account for this dependence is the use of the so-called effective medium

\footnotetext{
* To whom correspondence should be addressed: j.sancho.parramon@ gmail.com
} 
theories (EMTs), which calculate the optical constants of a mixture in terms of the optical constants of the mixing materials and their volume fractions [9, 10]. These theories have been widely used to compute the compositional dependence of the refractive index for different composites [11,12]. On the other hand, the applicability of these theories to compute the absorption coefficient is often overlooked. Nevertheless, the compositional dependence of the absorption coefficient can be relevant, since the absorption edge defines the spectral range in which an optical coating will be transparent. Even the determination of very small absorption values is important as it may limit the radiation resistance of a coating [6].

In this paper we study the validity of EMTs for photon energies around the absorption onset, where absorption is weak. In the second section we analyze theoretically the behaviour of EMTs in weak absorption ranges. Then, we study the case of $\mathrm{Nb}_{2} \mathrm{O}_{5}-\mathrm{SiO}_{2}$ composite thin films performing the optical characterization of a set of mixtures by inversion of experimental measurements. The optical constants of the mixture films are compared with the values computed using EMTs, enabling to evaluate the validity of these theories for both, refractive index and absorption coefficient. The limitations of EMTs are discussed in terms of the theoretical analysis of the second section.

\section{Theory}

The aim of EMTs is to calculate the effective dielectric function $\left(\varepsilon_{e f f}\right)$, that describes the optical behavior of a material mixture. The effective dielectric function is calculated in terms of the dielectric function of the mixing materials $\left(\varepsilon_{1}\right.$ and $\left.\varepsilon_{2}\right)$ and their geometrical arrangement. Over the last century several EMTs have been proposed, being the Bruggeman model among the most successful [13]:

$$
(1-f) \frac{\varepsilon_{1}-\varepsilon_{e f f}}{\varepsilon_{1}+2 \varepsilon_{e f f}}+f \frac{\varepsilon_{2}-\varepsilon_{e f f}}{\varepsilon_{2}+2 \varepsilon_{e f f}}=0,
$$

where $f$ is the volume fraction in the mixture of the component with dielectric function $\varepsilon_{2}$. Another commonly applied model is the Maxwell-Garnett equation [14]:

$$
\frac{\varepsilon_{e f f}-\varepsilon_{1}}{\varepsilon_{e f f}+2 \varepsilon_{1}}=f \frac{\varepsilon_{2}-\varepsilon_{1}}{\varepsilon_{2}+2 \varepsilon_{1}}
$$

Contrary to the Bruggeman model, here the mixing materials are treated in an unsymmetrical way, with the material with dielectric function $\varepsilon_{1}$ considered as host and the one with dielectric function $\varepsilon_{2}$ playing the role of inclusions. Besides these two most widely used models, other EMTs have been derived and successfully applied in some cases, like the one proposed by Looyenga [15]:

$$
\varepsilon_{e f f}^{1 / 3}=(1-f) \varepsilon_{1}^{1 / 3}+f \varepsilon_{2}^{1 / 3}
$$

All these effective medium theories assume that the composite is made up of phases of the mixing materials, having dimensions that are small compared to the wavelength of the interacting radiation and large enough to have their own electromagnetic behaviour. The difference between EMTs is the way how the interaction among the composing phases is taken into account. In this framework, Bergman demonstrated [16] that a spectral representation for any EMT can be found:

$$
\varepsilon_{\text {eff }}=\varepsilon_{1}\left(1-f \int_{0}^{1} \frac{g(u)}{t-u} d u\right) \text { where } t=\frac{\varepsilon_{1}}{\varepsilon_{1}-\varepsilon_{2}}
$$


where the function $g(u)$ is the so-called spectral density function. This formalism explicitly separates the influence of the dielectric function of the mixing materials $(t)$ from the specific topology of the mixture, that defines the interaction among the composing phases $(g(u))$. All the above described EMTs follow the spectral representation (4), having different spectral densities [17]. For instance, in the Maxwell-Garnett theory the spectral density is a delta function. From a mathematical point of view, the spectral density is a positive function defined in the range [0-1] and fulfils the following sum rules:

$$
\int_{0}^{1} g(u) d u=1 \quad \text { and } \quad \int_{0}^{1} u \cdot g(u) d u=\frac{1-f}{3}
$$

being the second expression valid only for isotropic media. Although in the Bergman representation one material is considered as host $\left(\varepsilon_{1}\right)$ and the other as inclusions $\left(\varepsilon_{2}\right)$, it is possible to reverse their roles by taking the appropriate spectral density function [18].

In order to analyze the behaviour of EMTs in weak absorption regions, we shall assume that only one material is absorbing $\left(\varepsilon_{I}=\varepsilon_{I R}+\mathrm{i} \varepsilon_{I I}\right.$ and $\left.\varepsilon_{2}=\varepsilon_{2 R}\right)$. In this case, the real $\left(\varepsilon_{\text {effR }}\right)$ and imaginary $\left(\varepsilon_{e f f I}\right)$ part of the effective dielectric function can be expressed as:

$$
\begin{aligned}
& \varepsilon_{\text {effR }}=\varepsilon_{1 R}\left(1-f \int_{0}^{1} \frac{g(u)}{\left(t_{R}-u\right)^{2}+t_{I}^{2}}\left(t_{R}-u\right) d u\right)-f \varepsilon_{1 I} \int_{0}^{1} \frac{g(u)}{\left(t_{R}-u\right)^{2}+t_{I}^{2}} t_{I} d u \\
& \varepsilon_{\text {effI }}=\varepsilon_{1 I}\left(1-f \int_{0}^{1} \frac{g(u)}{\left(t_{R}-u\right)^{2}+t_{I}^{2}}\left(t_{R}-u\right) d u\right)+f \varepsilon_{1 R} \int_{0}^{1} \frac{g(u)}{\left(t_{R}-u\right)^{2}+t_{I}^{2}} t_{I} d u
\end{aligned}
$$

with $t_{R}$ and $t_{I}$ standing for the real and imaginary part of $t$. In the case of weak absorption, we can assume $\varepsilon_{1 R}, \varepsilon_{2 R}$ and $\left|\varepsilon_{1 R}-\varepsilon_{2 R}\right| \gg>\varepsilon_{1 I}$, so $t_{R}$ and $t_{I}$ can be approximated as:

$$
t_{R} \approx \frac{\varepsilon_{1 R}}{\varepsilon_{1 R}-\varepsilon_{2 R}} \quad t_{I} \approx \frac{-\varepsilon_{1 I} \varepsilon_{2 R}}{\left(\varepsilon_{1 R}-\varepsilon_{2 R}\right)^{2}}
$$

For dielectric materials both, $\varepsilon_{1 R}, \varepsilon_{2 R}$ are positive so $\left|t_{\mathrm{R}}\right|>1$ if $\varepsilon_{1 R}>\varepsilon_{2 R}$. If $\varepsilon_{2 R}>\varepsilon_{1 R}$, we can exchange the roles of $\varepsilon_{I R}$ and $\varepsilon_{2 R}$ by taking the proper spectral density function as mentioned before, so we have $\left|t_{\mathrm{R}}\right|>1$. Assuming that $\left|t_{\mathrm{R}}-u\right|>>\left|t_{1}\right|$, for all the integration range in (6) and (7) $(u \in[0,1])$, the real and imaginary part of the effective dielectric function can be expressed as:

$$
\begin{array}{r}
\varepsilon_{\text {effR }} \cong \varepsilon_{1 R}\left(1-f \frac{\left(\varepsilon_{1 R}-\varepsilon_{2 R}\right)}{\varepsilon_{1 R}} G_{A}\right) \\
\varepsilon_{\text {effI }} \cong \varepsilon_{1 I}\left(1-f \frac{\left(\varepsilon_{1 R}-\varepsilon_{2 R}\right)}{\varepsilon_{1 R}} G_{A}-f \frac{\varepsilon_{2 R}}{\varepsilon_{1 R}} G_{B}\right)
\end{array}
$$

with:

$$
G_{A}=\int_{0}^{1} \frac{g(u)}{1-u / t_{R}} d u \quad G_{B}=\int_{0}^{1} \frac{g(u)}{\left(1-u / t_{R}\right)^{2}} d u
$$


where terms of $\varepsilon_{1 I}^{2}$ and higher orders have been neglected. These expressions show that the real part of $\varepsilon_{\text {eff }}$ depends on $\varepsilon_{1 R}$ and $\varepsilon_{2 R}$ but not on $\varepsilon_{1 I}$. On the contrary, the imaginary part of $\varepsilon_{e f f}$ depends not only on $\varepsilon_{1 I}$ but also on $\varepsilon_{1 R}$ and $\varepsilon_{2 R}$. This asymmetric behaviour is illustrated in figure 1. The influence of variations of $\varepsilon_{1 I}$ on $\varepsilon_{\text {effR }}$ (figure 1.a) and of $\varepsilon_{2 R}$ on $\varepsilon_{\text {eff }}$ (figure 1.b) are shown, using typical values for the dielectric function of $\mathrm{TiO}_{2}$ and $\mathrm{SiO}_{2}$ in weak absorption regions. Regardless the EMT applied, $\varepsilon_{2 R}$ has a remarkable effect over $\varepsilon_{\text {effl }}$ and the influence of $\varepsilon_{1 I}$ on $\varepsilon_{e f f R}$ is negligible for small values of $\varepsilon_{11}$.
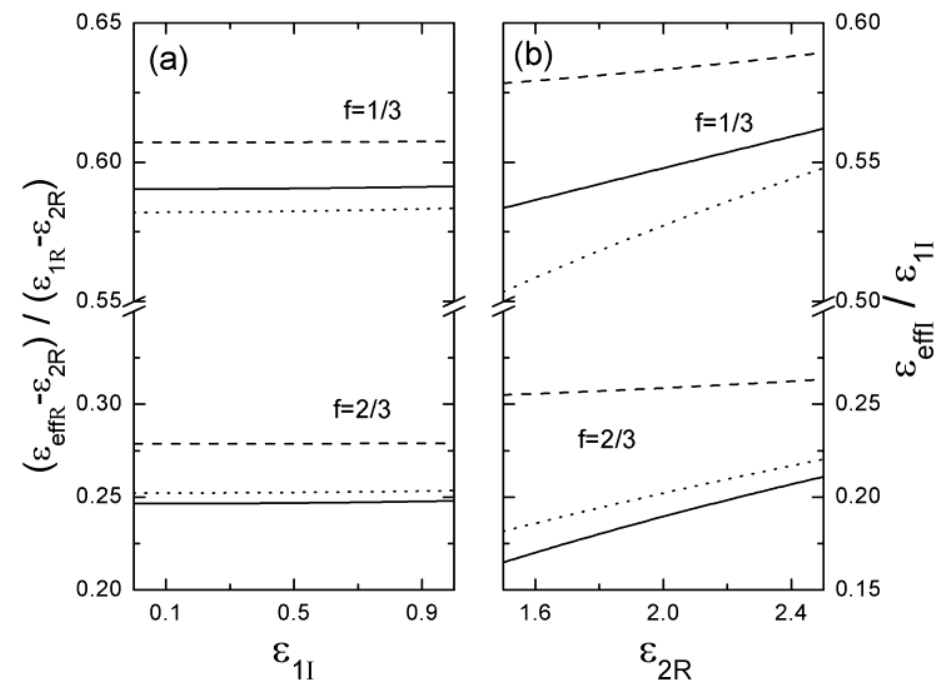

Figure 1. Influence of variations of $\varepsilon_{1 I}$ on $\varepsilon_{\text {effR }}$ (a) and of $\varepsilon_{2 R}$ on $\varepsilon_{\text {eff }}$ (b) for two different compositions $(f=1 / 3$ and 2/3) using Bruggeman (solid line), Maxwell-Garnett (dashed line) and Looyenga (dotted line) models for a mixture of materials with $\varepsilon_{1}=6.7+i \quad 0.5$ and $\varepsilon_{2}=2.13$.

In addition to expressions (9)-(10), it is possible to make a series expansion in powers of $t_{R}$ in the integrals in (11). Using the sum rules (5), the first two terms of these series expansion are independent of the spectral density function and approximate expressions for the effective dielectric function can be formulated as (for $\left|t_{R}\right|>>1$ ):

$$
\begin{aligned}
& \varepsilon_{\text {effR }} \approx(1-f) \varepsilon_{1 R}+f \varepsilon_{2 R}-\frac{f(1-f)}{3} \frac{\left(\varepsilon_{1 R}-\varepsilon_{2 R}\right)^{2}}{\varepsilon_{1 R}} \\
& \varepsilon_{\text {effI }} \approx\left(1-f-\frac{f(1-f)}{3} \frac{\left(\varepsilon_{1 R}^{2}-\varepsilon_{2 R}^{2}\right)}{\varepsilon_{1 R}^{2}}\right) \varepsilon_{1 I}
\end{aligned}
$$

These expressions show that in the case when the dielectric functions of the mixing materials are close, $\varepsilon_{e f f}$ becomes nearly independent of the spectral density, i.e. all EMTs are equivalent. This result is also illustrated in figure 1.b: as the value of $\varepsilon_{2 \mathrm{R}}$ increases, $t_{\mathrm{R}}$ grows and the discrepancies among $\varepsilon_{e f f}$ calculated by different EMTs decrease. It should be mentioned that this result is not limited to materials with weak absorption, as a series expansion of (4) in powers of $t$ can be also performed and provided that $|t|>>1 \varepsilon_{\text {eff }}$ becomes independent on $g(u)$ as well [17]. In that case, however, expressions (12) and (13) do not hold since $\varepsilon_{\text {effR }}$ may depend also on $\varepsilon_{11}$. It must be 
mentioned that for strong absorption regions, different EMTs, lead to different compositional dependences of the real and imaginary parts of the dielectric function [19].

If, instead of the dielectric function, we use the refractive index $(n)$ and the absorption coefficient $(\alpha)$ as optical constants, in weak absorption regions they are connected to $\varepsilon$ as:

$$
\begin{aligned}
& n=\sqrt{\frac{1}{2}\left(\sqrt{\varepsilon_{r}^{2}+\varepsilon_{i}^{2}}+\varepsilon_{r}\right)} \cong \sqrt{\varepsilon_{r}} \\
& \alpha=\frac{4 \pi}{\lambda} \sqrt{\frac{1}{2}\left(\sqrt{\varepsilon_{r}^{2}+\varepsilon_{i}^{2}}-\varepsilon_{r}\right)} \cong \frac{2 \pi \varepsilon_{i}}{\lambda n}
\end{aligned}
$$

where $\lambda$ is the wavelength of the interacting radiation and the dielectric constant of vacuum has been taken as unitary. Therefore, the conclusions for $\varepsilon_{\text {effR }}$ and $\varepsilon_{\text {eff }}$ in weak absorption regimes are also applicable to the refractive index and absorption coefficient: $n$ will depend only on the real part of the dielectric function, while $\alpha$ will depend on both real and imaginary parts.

\section{Experimental}

A set of $\mathrm{Nb}_{2} \mathrm{O}_{5}-\mathrm{SiO}_{2}$ composite layers was deposited on fused silica (Suprasil) substrates by coevaporation in a Leybold Syrius Pro 1100 system equipped with two electron beam guns and Advanced Plasma source [20]. This combination of materials was used for the design, deposition and characterization of hybrid coatings for antireflection purposes [21-23]. The composition of the mixtures was controlled by adjusting the deposition rates of both materials. Thus, mixtures with $\mathrm{Nb}_{2} \mathrm{O}_{5}: \mathrm{SiO}_{2}$ volume ratios of 80:20, 65:35, 50:50 35:65 and 20:80 were produced. Single layer of $\mathrm{Nb}_{2} \mathrm{O}_{5}$ and $\mathrm{SiO}_{2}$ were also deposited. The layer thickness was approximately $500 \mathrm{~nm}$ in all cases.

Reflectance and transmittance measurements at near-normal incidence were performed in the range 200-900 nm with a Perkin Elemer Lambda 900 spectrophotometer. In addition, structural characterization of the layers was carried out using different techniques (X-ray diffraction, transmission electron microscopy and infrared spectroscopy). The results of the structural characterization have been partially reported in [24], showing that the composites consist of a homogeneous mixture of $\mathrm{SiO}_{2}$ and $\mathrm{Nb}_{2} \mathrm{O}_{5}$ at molecular level. Analysis of measurements was carried out with the NKD optical characterization software package [25].

\section{Results}

\subsection{Optical characterization by direct data inversion}

For the optical characterization of the mixture films, we follow a two-step procedure. First, we determine the optical constants from the reflectance and transmittance data using a single wavelength algorithm [26], i.e. the refractive index and the absorption coefficient of the layer are extracted from direct data inversion at each measured wavelength. In a second step, the observed dependence of the optical constants with the wavelength is fitted to a suitable dispersion model. In the short wavelength part of the spectra, the transmittance spectra of the samples drop to zero due to the film absorption. In this case the extraction of $n$ and $\alpha$ from reflectance measurements only is not reliable. Therefore, the experimental data is inverted just in spectral regions with transmittance above a defined value $(\geq 0.5 \%)$.

The results of the direct data inversion for the refractive index and absorption coefficient are shown in figures 2 and 3. The refractive index of the mixtures increases with the content of 
$\mathrm{Nb}_{2} \mathrm{O}_{5}$. In general, the dependence of the refractive index with the energy is smooth, except for the wavelengths in which irregular dispersion is observed. Such discrepancies are caused by the multiplicity of solutions especially at wavelengths of spectral maxima and minima due to experimental error [27]. Apart from these discrepancies, the dispersion of the refractive index with the photon energy $(E)$ can be well described by the Cauchy expression:

$$
n(E)=n_{0}+n_{1} E^{2}+n_{2} E^{4}
$$

Fitting of refractive index dispersion to equation 16 is shown in figure 2.

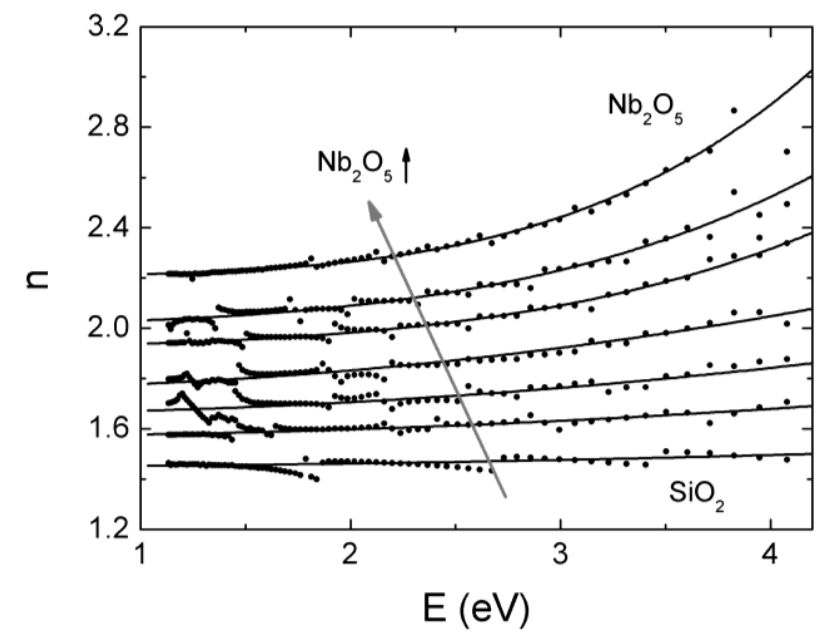

Figure 2. Dispersion of the refractive index for the $\mathrm{Nb}_{2} \mathrm{O} 5, \mathrm{SiO}_{2}$ and $\mathrm{Nb}_{2} \mathrm{O}_{5}: \mathrm{SiO}_{2}$ films with volume ratios 80:20, 65:35, 50:50, 35:65, 20:80 from inversion of experimental data (dots) and corresponding fits to the Cauchy expression (solid line).

Regarding the absorption properties, all samples show an absorption front that is shifted towards higher energies as the content of $\mathrm{SiO}_{2}$ increases (figure 3). For the pure $\mathrm{SiO}_{2}$ layer, no absorption is observed in the whole spectral range of measurements. The absorption front shows two different absorption regions. At low energies, the logarithm of the absorption coefficient has a linear dependence with the photon energy. Such dependence is related to electronic transitions between localized and extended electronic states and is usually described with the Urbach expression [28]:

$$
\alpha(E)=\alpha_{0} \exp \left(E / E_{\mu}\right)
$$

in which $E_{\mu}$ is the Urbach energy that is associated to structural and thermal disorder. At higher energies, the absorption coefficient follows the Tauc expression [29]:

$$
\alpha(E)=B\left(E-E_{g}\right)^{2} / E
$$

that describes absorption related to electronic transitions between valence and conduction band. $E_{g}$ is the Tauc gap and represents the energy onset for interband transitions, i.e. distance between valence and conduction band. $B$ is a parameter connected to the optical transition matrix. Instead of using two different expressions for describing the absorption in the whole measurement range, a more compact picture can be obtained by using the model proposed by O'Leary et al. [30]. In their work, they empirically model the electron density of states for the conduction $\left(N_{C}\right)$ and valence band $\left(N_{V}\right)$ accounting for both, extended states and the tail of localized states: 


$$
\begin{gathered}
N_{C}(E)=A_{C} \begin{cases}\sqrt{E-V_{C}}, & E \geq V_{c}+\frac{\gamma_{C}}{2} \\
\sqrt{\frac{\gamma_{C}}{2}} \exp \left(-\frac{1}{2}\right) \exp \left(\frac{E-V_{C}}{\gamma_{C}}\right), & E<V_{c}+\frac{\gamma_{C}}{2}\end{cases} \\
N_{V}(E)=A_{V} \begin{cases}\sqrt{\frac{\gamma_{V}}{2}} \exp \left(-\frac{1}{2}\right) \exp \left(\frac{V_{V}-E}{\gamma_{V}}\right), & E \geq V_{V}-\frac{\gamma_{V}}{2} \\
\sqrt{V_{V}-E}, & E<V_{V}-\frac{\gamma_{V}}{2}\end{cases}
\end{gathered}
$$

Here $V_{C}$ and $V_{V}$ define the conduction and valence band disorderless band edge, and $\gamma_{C}$ and $\gamma_{V}$ are the breadth of the conduction and valence band tail. $A_{C}$ and $A_{V}$ are energy independent constants. The absorption coefficient can be then calculated as:

$$
\alpha(E)=D^{2}(E) J(E)=\frac{A}{E} \int_{-\infty}^{\infty} N_{C}(E) N_{V}\left(E-E_{0}\right) d E
$$

in which $D^{2}(E)$ is the optical transition matrix element and $J(E)$ the joint density of states. We can further assume that $D^{2}(E)$ presents an inverse dependence with the energy as suggested in [30]. Analytical expressions for the integral in equation (21) are given in [30]. For the sake of simplicity we have assumed $\gamma_{C}=\gamma_{V} \equiv \gamma$ and defined $E_{0} \equiv V_{C}-V_{V}$. Fits of the absorption coefficient dispersion to this model are shown in figure 3. The optimum values of the parameters are summarized in Table 1.

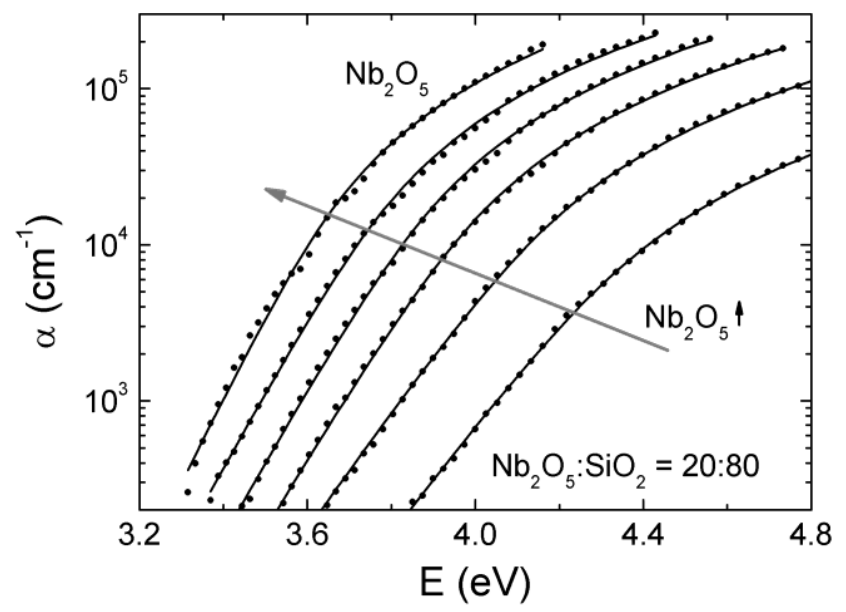

Figure 3. Dispersion of the absorption coefficient for the $\mathrm{Nb}_{2} \mathrm{O} 5$ and $\mathrm{Nb}_{2} \mathrm{O}_{5}: \mathrm{SiO}_{2}$ films with volume ratios 80:20, 65:35, 50:50, 35:65, 20:80 from inversion of experimental data (dots) and corresponding fits to O'Leary et al. model (solid line).

Table 1 Parameters of O'Leary et al. model describing dispersion of the absorption coefficient

\begin{tabular}{c|ccc}
\hline $\begin{array}{c}\text { Mixture } \\
\text { composition }\end{array}$ & $A\left(\mathrm{eV} \cdot \mathrm{cm}^{-1}\right)$ & $\gamma(\mathrm{meV})$ & $E_{0}(\mathrm{eV})$ \\
\hline $\mathrm{Nb}_{2} \mathrm{O}_{5}$ & $(4.30 \pm 0.40) \cdot 10^{6}$ & $67.3 \pm 3.0$ & $3.529 \pm 0.017$ \\
$80: 20$ & $(3.69 \pm 0.17) \cdot 10^{6}$ & $75.5 \pm 1.6$ & $3.634 \pm 0.010$ \\
$65: 35$ & $(3.22 \pm 0.14) \cdot 10^{6}$ & $78.2 \pm 1.4$ & $3.729 \pm 0.010$ \\
\hline
\end{tabular}




\begin{tabular}{c|ccc}
\hline $50: 50$ & $(2.48 \pm 0.08) \cdot 10^{6}$ & $82.9 \pm 1.2$ & $3.823 \pm 0.008$ \\
$35: 65$ & $(1.82 \pm 0.06) \cdot 10^{6}$ & $96.8 \pm 1.1$ & $3.974 \pm 0.009$ \\
$20: 80$ & $(9.13 \pm 0.3) \cdot 10^{5}$ & $104.0 \pm 1.5$ & $4.139 \pm 0.010$ \\
$\mathrm{SiO}_{2}$ & - & - & - \\
\hline
\end{tabular}

\subsection{Comparison with effective medium theories}

Once the optical constants for the mixture films are known, it is possible to evaluate the validity of effective medium theories. For this purpose, we take the optical constants determined from measurements of the pure $\mathrm{Nb}_{2} \mathrm{O}_{5}$ and $\mathrm{SiO}_{2}$ layers and for each sample the effective optical constants of the mixture are computed using the expression (1), (2) and (3). The volume fraction of the components is calculated from the deposition rates. The comparison between the optical constants inferred from experimental measurements and those calculated using the Bruggeman formula is shown in figures 4 and 5. The results for the Maxwell-Garnett and Looyenga expressions are very close to that of Bruggeman. For the sake of clarity they are not plotted on the graphs. In the transparent region, there is a good agreement between the experimentally determined refractive index and the one calculated using EMTs, while in the region of weak absorption the discrepancies are higher, up to 5\%. For the absorption coefficient, however, the differences are much larger: in some cases the calculated absorption coefficient is two orders of magnitude higher than the experimental one.

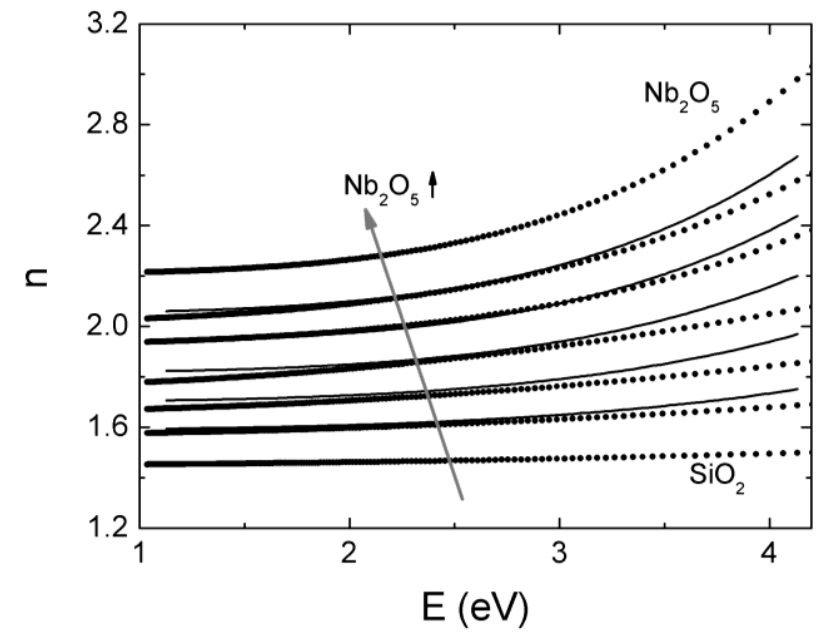

Figure 4. Comparison between the refractive index calculated using Bruggeman model (solid line) and the one experimentally determined and adjusted to the Cauchy expression (dots) 


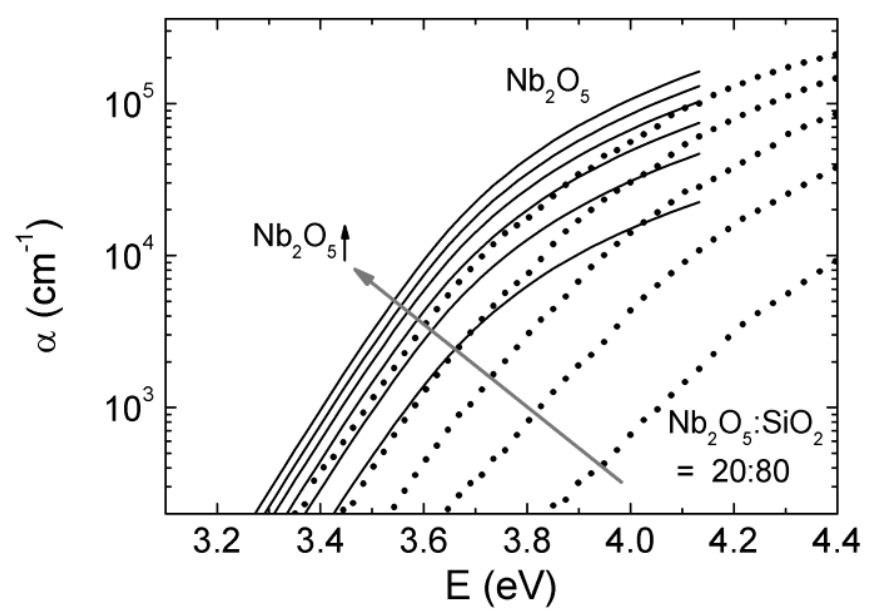

Figure 5. Comparison between the absorption coefficient calculated using Bruggeman model (solid line) and the one experimentally determined (dots)

\section{Discussion}

The two-steps procedure followed for inferring the optical constants from experimental measurements has been proved to be useful for a precise determination of all features of optical constants dispersion. Namely, we have also tried an alternative and more often employed approach: using a dispersion model to represent the film's optical constants and then find the optimal value of the model parameters by fitting the experimental data. If we use the Cauchy and O'Leary expressions as dispersion models, very good data fits are obtained, with optimal values of model parameters in agreement with those found using the two-step strategy. Nevertheless, we also achieved comparable data fits using the Tauc-Lorentz expression [31] as dispersion model. In opposition to the Cauchy and O'Leary expressions, the Tauc-Lorentz model neglects the absorption below the band-gap. Even though, in both cases it was obtained a similar merit function, i.e. total discrepancy between experimental and simulated data. The reason is that the below band-gap absorption influences the data only in a limited part of the spectral range and its overall weight on the merit function is small [32]. Thus, if no a-priori information is available, it may happen that the dispersion model chosen for data fitting leads to numerically good results, but also to an incomplete physical picture in part of the spectral range. In this framework, using the proposed two-step strategy can evidence specific features of optical constants dispersion and aid in the choice of the most proper dispersion model. This issue is particularly important in the present case, in which weak absorption levels are studied for comparison with EMTs calculations.

The discrepancies for the absorption coefficient between experimental data and simulation using EMTs can be understood from the results of Section 2. For weak absorption, according to equations (10), (11) and (15), the value of $\alpha$ at each wavelength will be proportional to $\alpha$ of $\mathrm{Nb}_{2} \mathrm{O}_{5}$ scaled to a factor that will depend on $\varepsilon_{R}$ of $\mathrm{Nb}_{2} \mathrm{O}_{5}$ and $\mathrm{SiO}_{2}$. Since the dispersion of these $\varepsilon_{R}$ is much smaller than the dispersion of $\alpha$ of $\mathrm{Nb}_{2} \mathrm{O}_{5}$, this proportionality factor can be considered as wavelength-independent. Therefore, EMTs cannot predict any change of the $\gamma$ or $E_{0}$ parameters of equation (21). This issue can be seen in the simulation in figure 5 in which all lines are parallel, i.e., having the same $\gamma$ for all mixtures as for $\mathrm{Nb}_{2} \mathrm{O}_{5}$. On the contrary, the situation for the refractive index is significantly better because in weak absorption regions, $\varepsilon_{\text {effR }}$ is unaffected by $\varepsilon_{11}$, according to equations (9) and (11). Finally, since the values of $|t|$ in the studied spectral range are between 1.4 and 1.9, the results are close to equations (12) and (13). Therefore, using different EMTs similar computed effective optical constants are obtained. 
In order to understand the failure of EMTs to describe the absorption properties of mixtures in weak absorption ranges properly, it must be recalled that EMTs assume that the materials are present in the mixture as two separated phases, with sizes large enough to have the same dielectric function locally as the bulk material. This is not the case in the mixtures studied in this work, in which the materials are well mixed at molecular level and therefore a clear phase separation does not exist [24]. However, failing to fulfil this assumption required in EMTs seems to be more critical for the absorption coefficient than for the refractive index. Other authors also reported a good agreement of EMTs for the refractive index of mixtures that showed no phase separation [11, 12]. The reason should be found in the fact that the strong dispersion of the absorption coefficient in weak absorption regions is tightly connected to the sample microstructure ( $E_{0}$ is the energy separation between bands and $\gamma$ is associated to the presence of tail states due to disorder). However, the refractive index shows a smaller dispersion in weak absorption regions and its value is primarily determined by the material density and less influenced by its microstructure. In any case, it can be seen in figure 4 that as the refractive index dispersion increases, going to higher photon energies, the error in calculation of EMTs for the refractive index becomes higher.

The observed behaviour of optical absorption in the studied $\mathrm{Nb}_{2} \mathrm{O}_{5}: \mathrm{SiO}_{2}$ films represents a common situation in composite thin films of interest for optical coatings. Namely, there are numerous cases consisting of mixtures of high and low refractive index in which the parameters that describe the dispersion of optical absorption in weak absorption ranges show a compositional dependence: $\mathrm{Y}_{2} \mathrm{O}_{3}: \mathrm{SiO}_{2}$ [33], $\mathrm{ZrO}_{2}: \mathrm{SiO}_{2}$ [34], $\mathrm{Gd}_{2} \mathrm{O}_{3}: \mathrm{SiO}_{2}$ [35], $\mathrm{MoO}_{3}: \mathrm{TeO}_{2}$ [35], $\mathrm{TiO}_{2}: \mathrm{SiO}_{2}$ [12, $36,37]$ or $\mathrm{Ta}_{2} \mathrm{O}_{5}: \mathrm{SiO}_{2}$ [38]. For most of these cases, results of structural characterization were presented, indicating that no clear phase separation is observable in the mixture. However, as already pointed out, EMTs were successfully used for describing the compositional dependence of the refractive index. On the other hand, in all these studies, the absorption properties of mixtures do not correspond to a simple rescaling of the absorption properties of the material with highest absorption, as EMTs predict. Therefore, the limitations of EMTs in weak absorption regions, exemplified here for $\mathrm{Nb}_{2} \mathrm{O}_{5}: \mathrm{SiO}_{2}$ mixtures, are supported by other studies.

The error in the absorption coefficient computed with EMTs can be critical for estimating the absorption threshold of a coating or its radiation resistance. For instance, for a coating to be used in $\mathrm{XeF}$ excimer laser optics $(351 \mathrm{~nm})$ using a 50:50 mixture of $\mathrm{Nb}_{2} \mathrm{O}_{5}: \mathrm{SiO}_{2}$, the measured absorption coefficient is about $250 \mathrm{~cm}^{-1}$. However, the EMTs predict a 10 times larger absorption coefficient. An alternative way to estimate the absorption of the coating is the parameterization of the compositional dependence of the dispersion parameters. It can be seen in table 1 that the O'Leary et al. model parameters follow a smooth variation with the composition. A simple linear interpolation between the dispersion parameter values of $\mathrm{Nb}_{2} \mathrm{O}_{5}$ and the 20:80 mixture (the absorption of $\mathrm{SiO}_{2}$ was zero in the studied spectral range and the O'Leary et al. model parameters could not be determined), leads to a value of the absorption coefficient within a $10 \%$ discrepancy in respect to the experimental value for the mentioned 50:50 mixture.

\section{Conclusions}

Using the Bergman spectral representation we have shown that all EMTs present a common behaviour in weak absorption regions: the real part of the effective dielectric function is independent of the imaginary part of the dielectric functions of the mixing materials, while the imaginary part of the effective dielectric function is basically a scaling of the imaginary part of the dielectric function of the material with absorption. When the dielectric functions of mixing materials are relatively close, all EMTs lead to the same results. 
The validity of EMTs has been tested on $\mathrm{Nb}_{2} \mathrm{O}_{5}: \mathrm{SiO}_{2}$ mixtures for energies around and below the band gap. The dispersion of the optical constants determined from experimental measurements, can be well described for all mixtures with the Cauchy expression for the refractive index and with the O'Leary et al. model the absorption coefficient. The comparison of the experimentally determined optical constants with those predicted by EMTs shows that the theories describe the compositional dependence of the absorption coefficient inadequately.

The fact that the absorption of the mixtures is calculated by EMTs as a simple scaling of the absorption of the mixing material disables any possibility to predict a compositional dependence of parameters like the band-gap energy or the band tail width. On the contrary, the absorption coefficient experimentally determined evidences a compositional dependence of these parameters. The errors in absorption coefficient predicted by EMTs are consequently very large and can induce a significant miscalculation of some crucial parameters of the coating like its transparency range or radiation resistance. In this framework, a more suitable approach to estimate the absorption coefficient of a mixture could be achieved by the parameterization of the compositional dependence of the dispersion model parameters.

\section{Acknowledgments}

The authors wish to acknowledge the Fraunhofrt Institut für Angewandte Optik und Feinmechanik (Jena, Germany), where the samples were deposited and characterized. VJ wants to thank the Fraunhofer Society for a Fraunhofer Fellowship. JSP wants to thank the Catalan Government for a "Beatriu de Pinos" postdoctoral grant.

\section{References}

[1] Willey R R 2002 Practical design and production of thin films (New York, Basel: Marcel Dekker)

[2] Janicki V, Leitel R, Wilbrandt S, Stenzel O, Gäbler D and Kaiser N 2005 Design of hybrid coatings composed of homogeneous layers and refractive index gradients Advances in Optical Thin Films II (Proceedings SPIE vol 5963) ed. C. Amra, N. Kaiser and H. A. Macleod IE pp 397404

[3] Rats D, Poitras D, Soro J M, Martinu L von Stebut J 1999 Surf. Coat. Technol. 111, 220-228

[4] Raymond M A, Larouche S, Zabeida O, Martinu L and Klemberg-Sapieha J E 2001 Tribological properties of PECVD optical coatings Proceedings of the $44^{\text {th }}$ Annual Technical Conference of Society of Vacuum Coaters ed. Society of Vacuum Coaters (Albuquerque, N. Mexico) pp 301-305

[5] Vernhes R, Zabeida O, Klemberg-Sapieha J E and Martinu L 2004 Appl. Opt. 43 97-103

[6] Ristau D, Schink H, Mittendorf F, Ebert J and Welling H 1988 Laser induced damage of dielectric systems with gradual interfaces at $1.064 \mu \mathrm{m}$ NIST Spec. Publ. (vol 775) pp. 414-426

[7] Poitras D, Larouche S and Martinu L 2002 Appl. Opt. 41 5249-5255

[8] Tikhonravov A V, Trubetskov M K, Amotchkina T V, Kokarev M A, Kaiser N, Stenzel O, Wilbrandt S, Gäbler D, 2006 Appl. Opt. 45 1515-1532

[9] Bosch S, Ferré-Borrull J, Leinfellner N and Canillas A 2000 Surf. Science 453 9-17

[10] Franta D and Ohlídal I, 2005, Opt. Comm. 248, 459-467

[11] Chen J -S, Chao S, Kao J -S, Niu H and Chen C-H 1996 Appl. Opt. 3590

[12] Wang X, Masumoto H, Someno Y and Hirai T 1999 Thin Solid Films 338 105-109

[13] Bruggeman D A 1935 Ann. Phys. 24 636-679

[14] Maxwell Garnett J C 1904 Philos. Trans. R. Soc. A 203 385-420

[15] Looyenga H 1965 Physica 31401

[16] Bergman D 1978 Physics Reports 43377 
[17] Theiss W 1993 Adv. in Sol. State Physics 33149

[18] Day A R and Thorpe M F 1999 J. Phys.: Condens. Matter 11 2251-2568

[19] Fujiwara H, Kooh Y, Rovira P I and Collins R W, 2000 Phys. Rew. B 61, 10832-10844

[20] Ehlers $\mathrm{H}$ et al. 2004 Ion assisted deposition processes; industrial network IntIon Advances in Optical Thin Films (Proceedings SPIE vol 5250) ed. C. Amra, N. Kaiser and H. A. Macleod pp 646-655

[21] Janicki V, Wilbrandt S, Stenzel O, Gäbler D, Kaiser N, Tikhonravov A, Trubetskov M and T. Amotchkina 2005 J. Opt. A: Pure Appl. Opt. 7 L9-L12

[22] Janicki V, Gäbler D, Wilbrandt S, Leitel R, Stenzel O, Kaiser N, Lappschies M, Görtz B, Ristau D, Rickers C and Vergöhl M 2006 Appl. Opt. 457851

[23] Janicki V, Sancho-Parramon J, Stenzel O, Lappschies M, Görtz B, Rickers C, Polenzky C and U. Richter. 2007 Appl. Opt. 46 6084-6091

[24] Sancho-Parramon J, Janicki V and Zorc H 2008 Thin Solid Films 516 5478-5482

[25] Bosch S, Ferré-Borrull J and Sancho-Parramon J 2001 Sol. Stat. Elec. 45703

[26] Stenzel O, Hopfe V and Klobes P 1991 J. Phys. D: Appl. Phys. 24 2088-2094

[27] Kutavichus V P, Filippov F and Huzouski V H 2006 Appl. Opt. 45 4547-4553

[28] Urbach F 1953 Phys. Rev. 921324

[29] Tauc J, Grigorivici R and Vancu A 1966 Phys. Status Solidi 15627

[30] O'Leary S K, Johnson S R and Lim P K 1997 J. Appl. Phys. 82 3334-3340

[31] Jellison Jr G E and Modine F A 1996 Appl. Phys. Lett. 69, 371-373 and 2137-2139

[32] Sancho-Parramon J, Bosch S and Canillas A 2006, App. Surf. Science 253 65-69

[33] Feldman A, Ying X and Farabaugh E N 1989, Appl. Opt. 28 5229-5232-4553

[34] Sahoo N K, Thakur S and Tokas R B 2006, J. Phys. D: Appl. Phys. 39 2571-2579

[35] Pal M, Tsujigami Y, Yoshikado A and Sakata H 2000, Phys. Stat. Sol. (a) 182 727-736

[36] Gracia F, Yubero F, Espinos J P, Holgado J P, Gonzalez Elipe R P and Girardeau T 2006, Surf. Interface Anal. 38 752-756

[37] Chrysicopoulou P, Davazoglou D, Trapalis C and Kordas G 2004, Journal of Material Science, 39 2835-2839

[38] Cevro M, 1995, Thin Solid Films, 258 91-103 